Gynecologic and

Obstetric Investigation
Gynecol Obstet Invest 2009;68:167-170

DOI: $\underline{10.1159 / 000230713}$
Received: November 20,2008

Accepted after revision: February 3, 2009

Published online: July 30, 2009

\title{
Thiazolidinediones as Therapy for Endometriosis: A Case Series
}

\author{
Molly B. Moravek ${ }^{\mathrm{a}}$ Elizabeth A. Ward ${ }^{\mathrm{a}}$ Dan I. Lebovic ${ }^{\mathrm{a}}$ b \\ Division of Reproductive Endocrinology and Infertility, Department of Obstetrics and Gynecology, \\ a University of Michigan, Ann Arbor, Mich. and b University of Wisconsin, Madison, Wisc., USA
}

\section{Key Words}

Endometriosis · Thiazolidinediones • Pelvic pain •

Rosiglitazone $\cdot$ Infertility

\begin{abstract}
Background: Current medical therapies for endometriosis result in delayed conception and have not been shown to provide any fertile benefit subsequent to treatment. Thiazolidinediones (TZDs) do not impede conception and have been shown to reduce endometriotic lesions in animal models; however, no studies have been performed in humans. The aim of this study was to provide preliminary data about the effectiveness of a TZD in treating endometriosis-related pain. Methods: Case series of women with endometriosis recruited from the University of Michigan as part of an openlabel prospective phase 2 a clinical trial. Participants were given rosiglitazone, $4 \mathrm{mg}$ daily, for 6 months. Subjective endometriosis symptoms were assessed using a modified Biberoglu and Behrman symptom severity scale and the McGill pain questionnaire. Results: Two of the 3 patients exhibited improvement in severity of symptoms and pain levels with a concurrent decrease in pain medication, while 1 experienced no change. Rosiglitazone was well tolerated by all patients. Conclusions: Combined with data gathered from studies in rats and nonhuman primates, the results from this study offer positive justification for using TZDs as a well-tolerated treatment for endometriosis that can address pain without impeding ovulation and without the need for add-back therapy.

Copyright $\odot 2009$ S. Karger AG, Basel
\end{abstract}

\section{Introduction}

Endometriosis is a relatively common gynecologic disease defined by the presence of extrauterine endometrial tissue. The endometrial tissue is most commonly found in the pelvis, in severe cases causing adhesions, scarring, and distorted pelvic anatomy; however, extrapelvic endometrial explants have been reported as well. Patients are generally of reproductive age, and may present with chronic pelvic pain, dysmenorrhea, dyspareunia, or subfertility. Although the prevalence of endometriosis in the general population is estimated to be about $10 \%[1]$, the prevalence in women with pelvic pain or subfertility is estimated to exceed $33 \%[2,3]$.

Several medical treatment regimens are utilized for endometriosis, including GnRH agonists, steroid contraceptives, progestins (oral and intrauterine device), and aromatase inhibitors [4-6]. However, side effect profile, cost, limited treatment duration, and relapse rate from these medications often make them undesirable or unrealistic [6]. Surgical treatment has similar limitations, particularly with respect to recurrence in pain over time [7]. Moreover, current medical options address pain symptoms while excluding attempts at conception. Present day medical therapies result in delayed conception and have not been shown to offer any fertile advantage subsequent to treatment [8].

Thiazolidinediones (TZDs) are a new class of drug that may show promise in treating endometriosis-induced pain while, contemporaneously, allowing these

\section{KARGER}

(ㄷ) 2009 S. Karger AG, Basel

Fax +41613061234

E-Mail karger@karger.ch

www.karger.com
Accessible online at:

www.karger.com/goi
Dan Lebovic

University of Wisconsin, Department of Ob/Gyn

Division of Reproductive Endocrinology and Infertility, H4/628 Clinical Science Center 600 Highland Avenue, Madison, WI 53792-3236 (USA)

Tel. +1 734262 3990, Fax +1 608262 9862, E-Mail lebovic@wisc.edu 
women the chance to conceive. Two TZDs, rosiglitazone and pioglitazone, were developed for the treatment of diabetes mellitus due to their ability to increase insulin sensitivity. While their mechanism is not fully understood, studies show they bind to and activate peroxisome proliferator-activated receptor- $\gamma$, found in many tissues throughout the body, including endometrial epithelial and stromal cells $[9,10]$. TZDs have been shown to inhibit both monocyte migration and peritoneal inflammatory cells in a mouse model [11-13], decrease chemokine and cytokine expression in endometriotic stromal cells $[9,10]$, and modulate angiogenesis [14]. Both rodent and baboon models of endometriosis have been utilized to demonstrate decreased endometriotic lesion burden with TZDs as compared with placebo [15-17]; however, no human trial currently exists in the literature.

The purpose of this study was to provide preliminary data on the role of TZDs as a treatment for endometriosis in humans. The following data comprise a limited series of patients with endometriosis treated with rosiglitazone for 6 months.

\section{Materials and Methods}

Women with endometriosis were recruited from the University of Michigan Endometriosis Center from July 2006 to May 2007 as part of an IRB-approved prospective phase 2a clinical trial (ClinicalTrials.gov, NCT00115661)

All patients had regular cycles, pelvic pain of $\geq 3$ months duration with a cyclical component and stage I-II endometriosis diagnosed by laparoscopy within the preceding 4 years. Stage IIIIV patients were not eligible for the study due to too many potential conflicting sources of pain in such patients, namely adhesive disease. The subjects were not pregnant, nonlactating and not using hormonal contraceptives for the previous month. They had no pre-existing liver dysfunction, malignancy, heart failure, diabetes or alternate causes of chronic pelvic pain. They all had normal white blood cell counts and fasting glucose-insulin ratios $(>4.5)$. None of the women had any psychiatric condition or substance abuse which would make compliance difficult. Subjects functioned as their own controls, as all measures were taken at baseline before initiating treatment.

Participants were instructed to start taking rosiglitazone (IND 72,318), 4 mg daily, for 6 months starting with their next menses. Subjective endometriosis symptoms were assessed using a modified Biberoglu and Behrman symptom severity scale (BB), which uses 3 -point rating scales $(0=$ no symptoms to $3=$ severe symptoms) to assess (1) dysmenorrhea by ability to work or conduct daily activities and pain medication requirements; (2) dyspareunia by interference with normal sexual activity, and (3) nonmenstrual pelvic pain by pain frequency and pain medication requirement [18]. The Short-Form McGill Pain Questionnaire was also used to evaluate the patients' perception of sensory and affective dimensions of their pain, as well as the degree of pain [19]. These surveys were administered just prior to starting the study drug, and at 3 and 6 months. Routine labs, including glucose, insulin, a complete blood count, liver enzymes, and a renal panel, were also drawn at these visits. Side effects were recorded at the 3- and 6month visits. Patients also kept a daily log of pain medication requirements starting a couple of weeks before taking the study drug through the end of the 6-month trial period.

\section{Results}

Six women (age 20-42 years) consented to participate in the study; 3 of them completed the full 6 -month trial (patients A, B, and C). Of the 3 women unable to complete the trial, one was deemed ineligible due to elevated liver enzymes and another patient was noncompliant secondary to multiple comorbidities and social issues, making it difficult for her to continue taking the study drug. The third woman ceased taking rosiglitazone only because of the concerns from the literature and subsequently the FDA. Patient D was only 3 months into the study when she was told the study was halted.

Patients A and B showed significant improvement in pain over the 6 months on rosiglitazone according to the McGill Pain Questionnaire, whereas patient C had no significant change in pain symptoms (fig. 1a). Additionally, patients $A$ and $B$ showed an improvement in symptoms as assessed by the modified $\mathrm{BB}$, while patient $\mathrm{C}$ again had no change (fig. $1 b$ ).

Patients A and B reported a drop in daily pain medication requirements from the start to the end of the study, and patient $\mathrm{A}$ also exhibited a transition from predominantly narcotics to more over-the-counter pain medications. Moreover, pain medication usage was recorded for patient $\mathrm{B}$ after discontinuing rosiglitazone, and demonstrated an increase back to pre-study levels. Patient C reported an inexplicable spike in pain medication requirements during the final month of the trial; however, she had decreased requirements from baseline in all of the preceding months.

One patient exhibited some weight loss during the 1st month on rosiglitazone, but then maintained a steady weight throughout the rest of the study. All other patients in this trial had no side effect related to rosiglitazone. Of note, however, some benefits of oral contraceptives were lost, such as acne control. Additionally, no change was observed in any of the routine laboratory studies from pre-study levels through the end of the study except for patient $\mathrm{C}$, who had one inexplicable spike in ALT during the 3rd month on the study drug, returning to normal levels at the next visit. In general, all patients on rosiglitazone tolerated the drug quite well. 

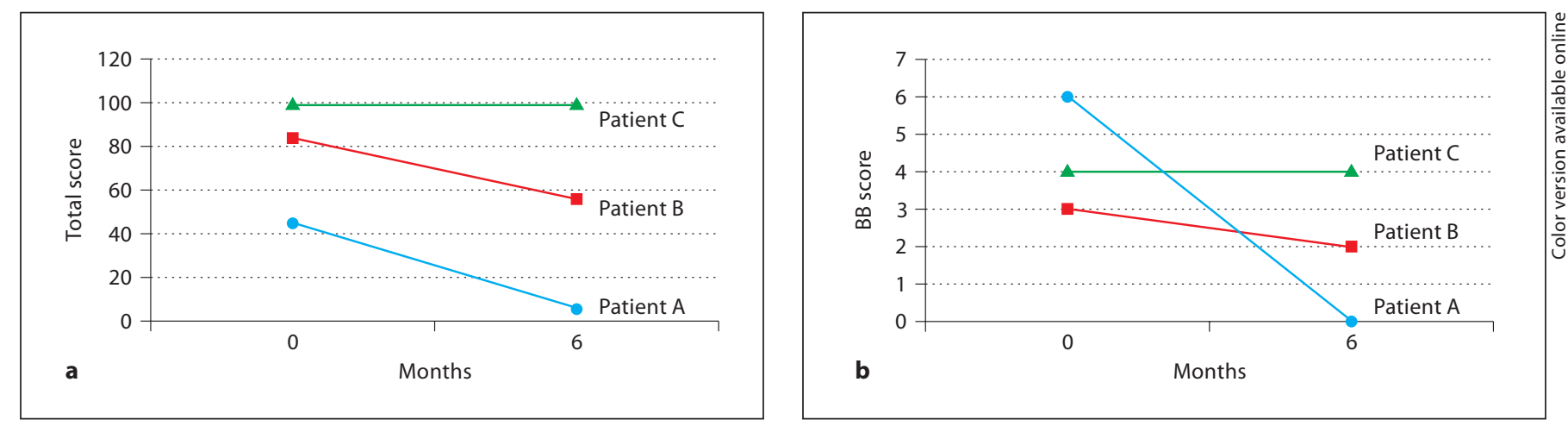

Fig. 1. Patient self-assessment of endometriosis symptoms before and after treatment with rosiglitazone. a Total score on McGill Pain Questionnaire at baseline and after 6 months on rosiglitazone (range: 0-150). b Modified BB symptom severity score at baseline and after 6 months of rosiglitazone (range: $0-9$ ).

Table 1. Medical treatment options for endometriosis demonstrating the comparative features of pioglitazone, a thiazolidinedione class drug

\begin{tabular}{llllll}
\hline & $\begin{array}{l}\text { Cost/month } \\
\text { USD }\end{array}$ & $\begin{array}{l}\text { Side effect } \\
\text { severity }^{\mathrm{a}}\end{array}$ & $\begin{array}{l}\text { Need for } \\
\text { add-back }\end{array}$ & $\begin{array}{l}\text { Pregnancy } \\
\text { category }\end{array}$ & $\begin{array}{l}\text { Conception } \\
\text { possible? }\end{array}$ \\
\hline Oral contraceptives & 60 & + & no & $\mathrm{X}$ & no \\
GnRH agonists & 600 & ++++ & yes & $\mathrm{X}$ & no \\
Depo-subQ provera 104 & 100 & ++++ & no & $\mathrm{X}$ & no \\
Mirena IUD & 10 & ++ & no & X & no \\
Aromatase inhibitors & 270 & ++ & yes & D & no \\
Pioglitazone & 175 & ++ & no & C & yes \\
\hline
\end{tabular}

Note the favorable side effect rating as well as pregnancy category for pioglitazone. Perhaps most importantly, and singular to this drug, pioglitazone may not hinder a woman's desire to conceive. Note, however, that pioglitazone is not an FDA-approved medicine for endometriosis.

a Side effect severity denoted by + (few) to ++++ (many) [26].

${ }^{\mathrm{b}}$ Add-back for bone preservation (i.e. estrogen).

\section{Discussion}

This is the first study looking at TZDs in humans suffering from endometriosis-induced pelvic pain. As seen in table 1, when compared with currently available therapeutic options, TZDs are a well-tolerated, affordable treatment for endometriosis that can address pain without the need for add-back therapy. Moreover, and unique to TZDs, there seems to be no impedance to ovulation. While other current treatment options, such as oral contraceptives, may have favorable prices or side effect profiles, they do not offer a chance at conception. Although the data for this study are certainly limited, this case series, combined with the data gathered from studies in rats and nonhuman primates, shows promise for the potential of using TZDs in endometriosis patients desiring the chance to conceive.

A major limitation to our trial was the number of patients and the limited course of therapy. In June 2007, the New England Journal of Medicine published a meta-analysis of rosiglitazone used for glycemic control wherein the authors concluded there was an approximately 50\% increased relative risk of myocardial ischemic events (absolute difference $<0.15 \%$ ) [20]. This information was widely reported in the lay press and, even though our participants were not diabetics we felt that the most prudent course of action was to suspend our trial. Because the study had to be stopped prematurely, the overall number 
of patients in this trial was limited and statistical analysis was prohibited. However, this study provides initial information to inform future clinical trials with larger participant recruitment. Additionally, while rosiglitazone has been given a black box warning label from the FDA due to an increased risk of cardiovascular side effects in heart failure patients, pioglitazone remains on the market and might be a viable substitute.

Since we are suggesting the possibility of using TZDs for endometriosis pain relief in conjunction with attempts to conceive, it must be understood that, under the current FDA safety classification scheme, TZDs are listed as a class $\mathrm{C}$ drug. There are limited studies on the potential reproductive toxicity induced by TZDs; however, animal studies show no sign of altered development and the rare human studies corroborate these findings [21, 22]. Furthermore, and in support of using such drugs in reproductive aged women, pioglitazone was used in a small trial as an ovulation induction agent in women with polycystic ovary syndrome. As opposed to rosiglitazone, pio- glitazone produces a better overall lipid profile - reductions in serum triglycerides and increases in high-density lipoprotein cholesterol levels [23, 24].

Due to the small number of participants in this trial, definitive conclusions would be premature; in fact, due to the background placebo effect with any medical therapy this may mitigate any decisive statement from case reports. For example, a recent endometriosis study using TNF- $\alpha$ antibodies had a 25\% overall placebo effect [25]. Nevertheless, the preliminary positive findings of this study utilizing TZD treatment warrant larger confirmatory studies. Future studies should assess more objective measures of the extent of endometriosis using laparoscopy.

\section{Acknowledgments}

We generously thank Dr. Daniel Szekely of the University of Michigan, Department of Obstetrics and Gynecology for referring study patients. Sources of financial support: $\mathrm{NIH}$ 5K23HD043952-02 (D.I.L.), Bayer Droegemueller Award (D.I.L.).

\section{References}

1 Eskenazi B, Warner ML: Epidemiology of endometriosis. Obstet Gynecol Clin North Am 1997;24:235-258.

-2 D'Hooghe TM, Debrock S, et al: Endometriosis and subfertility: is the relationship resolved? Semin Reprod Med 2003;21:243-254

3 Guo SW, Wang Y: The prevalence of endometriosis in women with chronic pelvic pain. Gynecol Obstet Invest 2006;62:121-130.

4 Petta CA, Ferriani RA, et al: Randomized clinical trial of a levonorgestrel-releasing intrauterine system and a depot GnRH analogue for the treatment of chronic pelvic pain in women with endometriosis. Hum Reprod 2005;20:1993-1998.

5 Treatment of pelvic pain associated with endometriosis. Fertil Steril 2006;86(suppl 5): S18-S27.

6 Mihalyi A, Simsa P, et al: Emerging drugs in endometriosis. Expert Opin Emerg Drugs 2006;11:503-524

-7 Surrey ES, Hornstein MD: Prolonged GnRH agonist and add-back therapy for symptomatic endometriosis: long-term follow-up. Obstet Gynecol 2002;99:709-719.

8 Hughes E, Brown J, et al: Ovulation suppression for endometriosis. Cochrane Database Syst Rev 2007;CD000155.

-9 Pritts EA, Zhao D, et al: PPAR-gamma decreases endometrial stromal cell transcription and translation of RANTES in vitro. J Clin Endocrinol Metab 2002;87:1841-1844.

10 Ohama Y, Harada T, et al: Peroxisome proliferator-activated receptor-gamma ligand reduced tumor necrosis factor-alpha-induced interleukin-8 production and growth in endometriotic stromal cells. Fertil Steril 2008; 89:311-317.

-11 Hornung D, Waite LL, et al: Nuclear peroxisome proliferator-activated receptors alpha and gamma have opposing effects on monocyte chemotaxis in endometriosis. J Clin Endocrinol Metab 2001;86:3108-3114.

12 Hornung D, Chao VA, et al: Thiazolidinedione inhibition of peritoneal inflammation. Gynecol Obstet Invest 2003;55:20-24.

13 Giudice LC, Kao LC: Endometriosis. Lancet 2004;364:1789-1799.

14 Giaginis C, Margeli A, et al: Peroxisome proliferator-activated receptor-gamma ligands as investigational modulators of angiogenesis. Expert Opin Investig Drugs 2007;16: 1561-1572.

15 Lebovic DI, Kir M, et al: Peroxisome proliferator-activated receptor-gamma induces regression of endometrial explants in a rat model of endometriosis. Fertil Steril 2004; 82(suppl 3):1008-1013.

16 Lebovic DI, Mwenda JM, et al: PPAR-gamma receptor ligand induces regression of endometrial explants in baboons: a prospective, randomized, placebo- and drug-controlled study. Fertil Steril 2007;88(suppl 4):1108-1119.

17 Demirturk F, Aytan H, et al: Effect of peroxisome proliferator-activated receptor-gamma agonist rosiglitazone on the induction of endometriosis in an experimental rat model. J Soc Gynecol Investig 2006;13:58-62.

$\checkmark 18$ Biberoglu KO, Behrman SJ: Dosage aspects of danazol therapy in endometriosis: short- term and long-term effectiveness. Am J Obstet Gynecol 1981;139:645-654.

19 Melzack R: The short-form McGill Pain Questionnaire. Pain 1987;30:191-197.

20 Nissen SE, Wolsk K: Effect of rosiglitazone on the risk of myocardial infarction and death from cardiovascular causes. N Engl J Med 2007;356:2457-2471.

21 Yaris F, Yaris E, et al: Normal pregnancy outcome following inadvertent exposure to rosiglitazone, gliclazide, and atorvastatin in a diabetic and hypertensive woman. Reprod Toxicol 2004;18:619-621.

22 Kalyoncu NI, Yaris F, et al: A case of rosiglitazone exposure in the second trimester of pregnancy. Reprod Toxicol 2005;19:563-564.

23 Derosa G, Cicero AF, et al: Effects of 1 year of treatment with pioglitazone or rosiglitazone added to glimepiride on lipoprotein (a) and homocysteine concentrations in patients with type 2 diabetes mellitus and metabolic syndrome: a multicenter, randomized, double-blind, controlled clinical trial. Clin Ther 2006;28:679-688.

24 Betteridge DJ: Effects of pioglitazone on lipid and lipoprotein metabolism. Diabetes Obes Metab 2007;9:640-647.

25 Koninckx PR: Anti-TNF- $\alpha$ treatment for deep endometriosis-associated pain: a randomized placebo-controlled trial. Hum Reprod 2008;9:2017-2023.

26 Vercellini P, Somigliana E, et al: Endometriosis: current and future medical therapies. Best Pract Res Clin Obstet Gynaecol 2008; 22:275-306. 